Vietnam Journal of Mechanics, NCST of Vietnam Vol. 23, 2001, No 3 (134 - 148)

\title{
STABILITY ANALYSIS OF STEEL FRAMES WITH SEMIRIGID CONNECTIONS AND SEMIRIGID CONNECTIONS WITH RIGIDZONES
}

\author{
Vu QUOC ANH \\ Faculty of Civil Engineering - Ha noi Architectural University
}

\begin{abstract}
To obtain an accurate insight into the behavior of most realistic steel frames, joint flexibility should be allowed for in the analysis since connection flexibility affects both force distribution and deformation in beams and columns of the frames. This paper proposes a method to establish the geometric stiffness matrix and stiffness matrix for semirigid beam element and semirigid beam element with rigidzones. The proposed method can also applied to analyse frame stability with rigid, semirigid and simple connections with rigidzones or without rigidzones. In addition, an approach that evaluates the effective length $\mu$ factor, the critical axial load of column in plane frames with rigid, semirigid, and simple connections is also presented.
\end{abstract}

\section{Introduction}

In the majority of civil engineering structures and building, the fabrication of a near fully rigid joint is expensive, impractical and economically unjustifiable in most cases. In reality, connections in steel frames are mostly semirigid and, consequently, the force and bending moment diagrams constructed under the rigid or pinned joined assumption contains a considerable error. The design based on these results will also lead to an inappropriate sizing of the members. In current engineering practice, the determination of the effective length factors of columns must be made in order to design semirigid frames to ensure accuracy. A major limitation of the column $\mu$ factor obtained from current methods is that they are not applicable to frames with semirigid connections and semirigid connections with rigidzones, but to perfectly rigid or simply connected frames. Therefore, there is a real need for a comprehensive and practical approach, preferably classical, for the stability analysis and calculation. of the effective length $\mu$ factor of each column in multistory multibay frames of any type of construction.

In the present paper, the governing equations for determining the column $\mu$ - factor for frames with semirigid connections and semirigid connections with rigidzones under various boundary conditions are derived based on the stability of semirigid frames.

The main objective of this paper is to present a stiffness matrix and geometric stiffness matrix of beam with semirigid connections and semirigid connections with rigidzones. The proposed matrices are applicable to the stability analyses of frames 
with rigid, semirigid, and simple connections with rigid zones or without rigidzones.

Recognizing the importance of connection flexibility and its effect on the performance of the structure, in various design codes for steel building such as the AISC [6] (1996), Eurocode-3 [7], and British Standard 5950 (1985) [8], the effects of joint flexibility are allowed to be accounted for in analysis [3]. The present study aims at developing a computer-oriented stability analysis for steel frame with rigid, semirigid and simple connections.

2. The stiffness matrix and geometric stiffness matrix of beam $\left[k_{e}\right]$, $\left[k_{\sigma}\right]$-Plane Frame

The linear elastic analysis assumes that the deformations are relatively small, and the equilibrium equations can be formulated with respect to initial geometry. When increasing applied loads cause significant changes in structure's geometry, the equilibrium and compatibility equations are nonlinear and the resulting stiffness matrix contains terms that are functions of axial forces and deformations. The stiffness matrix to represent this behavior must include the effect of geometric nonlinearity. In addition, for members with flexible connections and rigidzones at the ends, the stiffness matrix must be modified to account for the effect of connection flexibility and rigidzones. For stability analysis of plane frames with semirigid connections and rigidzones, the stiffness matrix and geometric stiffness matrix of beam with semirigid connections and semirigid connections with rigidzones will be established.

The well-known elastic force-displacement relationship [5], Fig. 1a, for prismatic beam without shearing deformation, is:

$$
\left[\begin{array}{l}
f_{1} \\
f_{2} \\
f_{3} \\
f_{4}
\end{array}\right]=\frac{E J}{L^{3}}\left[\begin{array}{cccc}
12 & 6 L & -12 & 6 L \\
6 L & 4 L^{2} & -6 L & 2 L^{2} \\
-12 & -6 L & 12 & -6 L \\
6 L & 2 L^{2} & -6 L & 4 L^{2}
\end{array}\right] \times\left[\begin{array}{l}
x_{1} \\
x_{2} \\
x_{3} \\
x_{4}
\end{array}\right] \quad \text { or, }\{f\}=\left[k_{e}\right]\{x\}
$$

In the case of a beam element with bending properties in which the deformed shape is assumed to be a cubic function due to the rotations $x_{2}$ and $x_{4}$ at the ends, additional moments $f_{2}$ and $f_{4}$ are developed. From reference [1] the forcedisplacement relationship is given by the following equation:

$$
\left[\begin{array}{l}
f_{1} \\
f_{2} \\
f_{3} \\
f_{4}
\end{array}\right]=\frac{P}{30 L}\left[\begin{array}{cccc}
36 & 3 L & -36 & 3 L \\
3 L & 4 L^{2} & -3 L & -L^{2} \\
-36 & -3 L & 36 & -3 L \\
3 L & -L^{2} & -3 L & 4 L^{2}
\end{array}\right] \times\left[\begin{array}{l}
x_{1} \\
x_{2} \\
x_{3} \\
x_{4}
\end{array}\right] \text { or, }\{f\}=\left\{k_{\sigma}\right\}\{x\}
$$

where $L$ is length of beam; $E$ - modulus of elasticity; $J$ - moment of inertia; $P$ axial force in beam; $\left[k_{e}\right]$ - stiffness matrix of bearn; $\left[k_{\sigma}\right]$ - geometric stiffness matrix 
of beam; $\{x\}$ - vector of nodal point displacements of beam; $\{f\}$ - vector of nodal point forces of beam;

In what follows we emphasize classical buckling analysis, which uses $\left[k_{\sigma}\right]$. One begins by applying to the structure a reference level of loading $\{f\}_{\text {ref }}$ and carrying out a standard linear static analysis to obtain membrane stresses in elements [1]. Hence, we generate a geometric stiffness matrix $\left[k_{\sigma}\right]_{\text {ref }}$ appropriate to $\{f\}_{\text {ref }}$. For another load level, with $\lambda$ a scalar multiplier,

$$
\left[k_{\sigma}\right]=\lambda\left[k_{\sigma}\right]_{\text {ref }} \text { when } \quad\{f\}=\lambda\{f\}_{\text {ref }} .
$$

Equations (2.3) imply that multiplying all loads $f_{i}$ in $\{f\}_{\text {ref }}$ by $\lambda$ also multiplies the intensity of the stress field by $\lambda$ but does not change the distribution of stresses. Then, since external loads do not change during an infinitesimal buckling displacement $\{d x\}$,

$$
\left([k]+\lambda_{c r}\left[k_{\sigma}\right]_{r e f}\right)\{x\}=\left([k]+\lambda_{c r}\left[k_{\sigma}\right]_{r e f}\right)\{x+d x\}=\lambda_{c r}\{f\}_{r e f} .
$$

Subtraction of the first equation from the second yields:

$$
\left([k]+\lambda_{\text {cr }}\left[k_{\sigma}\right]_{\text {ref }}\right)\{d x\}=\{0\}
$$

Equation (2.5) defines an eigenvalue problem whose lowest eigenvalue $\lambda_{c r}$ is associated with buckling. The critical or buckling load is, from Eq. (2.3),

$$
\{f\}_{c r}=\lambda_{c r}\{f\}_{\text {ref }} .
$$

The eigenvector $\{d x\}$ associated with $\lambda_{c r}$ defines the buckling mode. The magnitude of $\{d x\}$ is indeterminate. Therefore $\{d x\}$ identifies shape but not amplitude.

Critical load calculations that tell nothing about the history of the response of a structure to load but that often yield relatively quick estimates of the upper bound of resistance of a system can also be useful. And since current steel design standards use the effective length factors determined from the critical loads to design columns of frames [2], [VSD].

3. To create the stiffness matrix and geometric stiffness matrix of beam with semirigid connections $\left[k_{e s}\right],\left[k_{\sigma s}\right]$ - Plane frame

\subsection{To create the stiffness matrix of beam with semirigid connections, $\left[k_{e s}\right]$}

Structural model of beam with semirigid connections, Fig. 1, beam with semirigid connections and rigidzones, Fig. 3. 

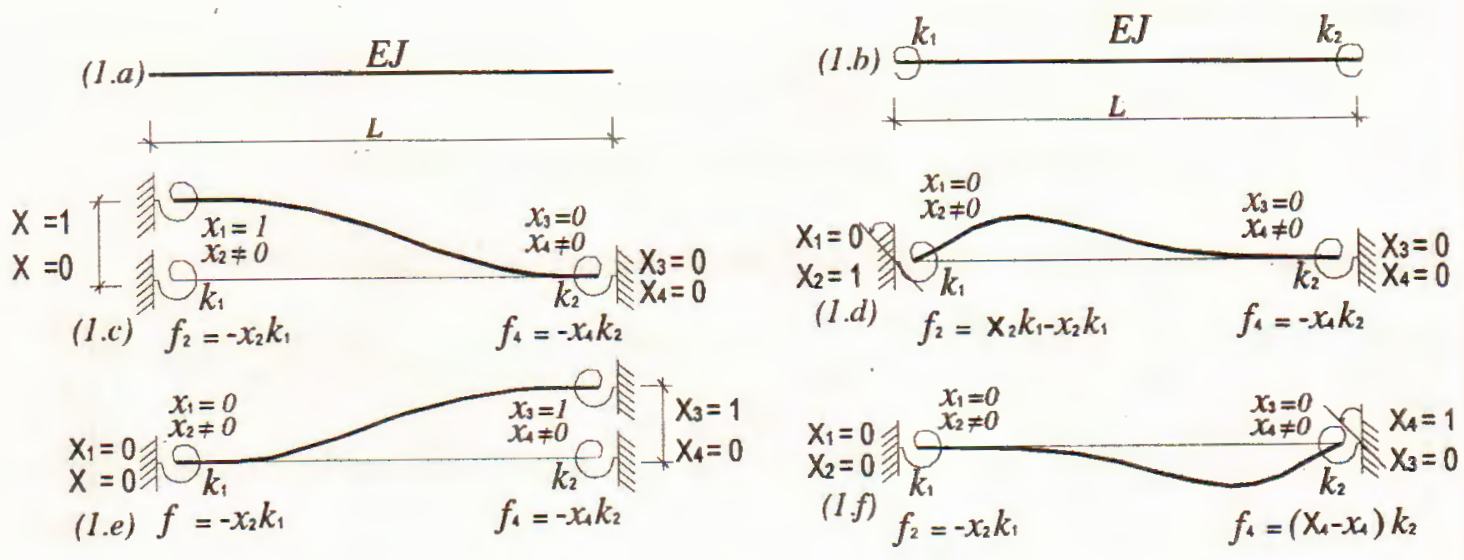

Fig. 1
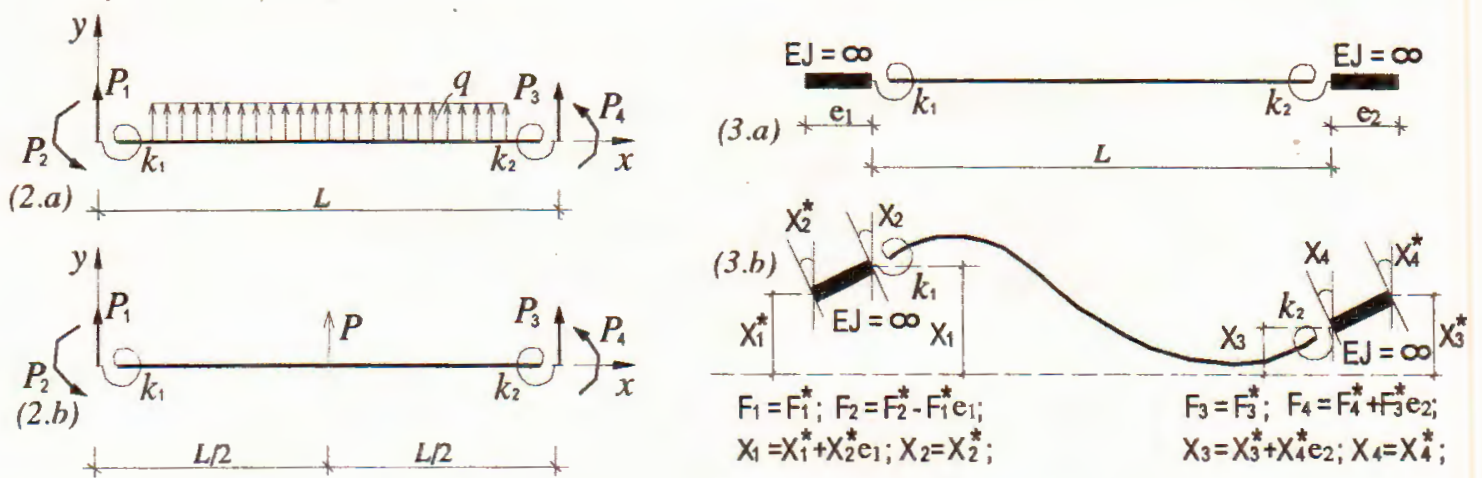

Fig. 2

Fig. 3

where: $\left[k_{e s}\right]$ stiffness matrix of beam with semirigid connections; $\{X\}$ vector of nodal point displacements of beam with semirigid connections; $\{F\}$ vector of nodal point forces of beam with semirigid connections; $e_{1}, e_{2}$ - length of rigidzones (rigid offset) of connections; $\left\{X^{*}\right\}$ - vector of nodal point displacements of beam with semirigid connections and rigidzones; $\left\{F^{*}\right\}$ - vector of nodal point forces of beam with semirigid connections and rigidzones; $k_{1} ; k_{2}$ - spring rotation factor of connections.

The elastic force deformation relationship, Fig. $1 \mathrm{~b}$, for a prismatic beam with semirigid connections, is:

$$
\left[\begin{array}{l}
F_{1} \\
F_{2} \\
F_{3} \\
F_{4}
\end{array}\right]=\left[\begin{array}{llll}
k_{11} & k_{12} & k_{13} & k_{14} \\
k_{21} & k_{22} & k_{23} & k_{24} \\
k_{31} & k_{32} & k_{33} & k_{34} \\
k_{41} & k_{42} & k_{43} & k_{44}
\end{array}\right] \times\left[\begin{array}{c}
X_{1} \\
X_{2} \\
X_{3} \\
X_{4}
\end{array}\right] \quad \text { or, }\{F\}=\left[k_{e s}\right]\{X\}
$$

The equilibrium equations of nodal point forces:

$$
\{F\}=\{f\} ; \quad\{F\}=\left[\begin{array}{llllll}
F_{1} & F_{2} & F_{3} & F_{4}
\end{array}\right]^{T} ; \quad\{f\}=\left[\begin{array}{llll}
f_{1} & f_{2} & f_{3} & f_{4}
\end{array}\right]^{T} .
$$

The first column of matrix $\left[k_{e s}\right]$ is created as follows 
Boundary conditions, Fig. 1c:

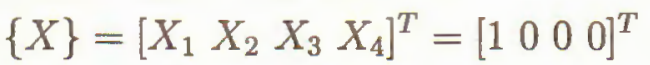

$$
\begin{aligned}
& f_{2}=-k_{1} x_{2} ; \quad f_{4}=-k_{2} x_{4} ; \quad x_{1}=1, \quad x_{3}=0 .
\end{aligned}
$$

To impose the boundary conditions into equations (2.1) that becomes:

$$
\left\{\begin{array} { l } 
{ \frac { 6 E L } { L ^ { 2 } } x _ { 1 } + \frac { 4 E J } { L } x _ { 2 } + \frac { 2 E J } { L } x _ { 4 } = - k _ { 1 } x _ { 2 } } \\
{ \frac { 6 E J } { L ^ { 2 } } x _ { 1 } + \frac { 2 E J } { L } x _ { 2 } + \frac { 4 E J } { L } x _ { 4 } = - k _ { 2 } x _ { 4 } }
\end{array} \Leftrightarrow \left\{\begin{array}{l}
\left(\frac{4 E J}{L}+k_{1}\right) x_{2}+\frac{2 E J}{L} x_{4}=-\frac{6 E J}{L^{2}} \\
\frac{2 E J}{L} x_{2}+\left(\frac{4 E J}{L}+k_{2}\right) x_{4}=-\frac{6 E J}{L^{2}}
\end{array}\right.\right.
$$

Solving equations (3.2) to find $x_{2} ; x_{4}$ :

$$
\begin{aligned}
& x_{2}=\frac{-6 E J\left(2 E J+k_{2} L\right)}{\left(12 E^{2} J^{2}+4 E J k_{2} L+4 L k_{1} E J+L^{2} k_{1} k_{2}\right) L} ; \\
& x_{4}=\frac{-6 E J\left(2 E J+k_{1} L\right)}{\left(12 E^{2} J^{2}+4 E J k_{2} L+4 L k_{1} E J+L^{2} k_{1} k_{2}\right) L},
\end{aligned}
$$

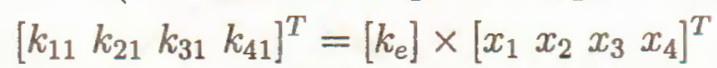

$$
\begin{aligned}
& {\left[\begin{array}{l}
k_{11} \\
k_{21} \\
k_{31} \\
k_{41}
\end{array}\right]=\left[\begin{array}{c}
\frac{12 E J}{L^{3}}-\frac{36 E^{2} J^{2}\left(2 E J+k_{2} L\right)+36 E^{2} J^{2}\left(2 E J+k_{1} L\right)}{L^{3}\left(12 E^{2} J^{2}+4 E J k_{2} L+4 L k_{1} E J+L^{2} k_{1} k_{2}\right)} \\
\frac{6 E J}{L^{2}}-\frac{24 E^{2} J^{2}\left(2 E J+k_{2} L\right)+12 E^{2} J^{2}\left(2 E J+k_{1} L\right)}{L^{2}\left(12 E^{2} J^{2}+4 E J k_{2} L+4 L k_{1} E J+L^{2} k_{1} k_{2}\right)} \\
-\frac{12 E J}{L^{3}}+\frac{36 E^{2} J^{2}\left(2 E J+k_{2} L\right)+36 E^{2} J^{2}\left(2 E J+k_{1} L\right)}{L^{3}\left(12 E^{2} J^{2}+4 E J k_{2} L+4 L k_{1} E J+L^{2} k_{1} k_{2}\right)} \\
\frac{6 E J}{L^{2}}-\frac{12 E^{2} J^{2}\left(2 E J+k_{2} L\right)+24 E^{2} J^{2}\left(2 E J+k_{1} L\right)}{L^{2}\left(12 E^{2} J^{2}+4 E J k_{2} L+4 L k_{1} E J+L^{2} k_{1} k_{2}\right)}
\end{array}\right]}
\end{aligned}
$$

The second column of matrix $\left[k_{e s}\right]$ is created as follows Boundary conditions, Fig. 1d:

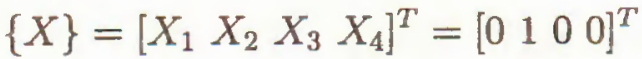

$$
\begin{aligned}
& f_{2}=k_{1}-k_{1} x_{2} ; \quad f_{4}=-k_{2} x_{4} ; \quad x_{1}=0, \quad x_{3}=0 .
\end{aligned}
$$

To impose the boundary conditions into equations (2.1) that becomes:

$$
\left\{\begin{array} { l } 
{ \frac { 4 E J } { L } x _ { 2 } + \frac { 2 E J } { L } x _ { 4 } = k _ { 1 } - k _ { 1 } x _ { 2 } } \\
{ \frac { 2 E J } { L } x _ { 2 } + \frac { 4 E J } { L } x _ { 4 } = - k _ { 2 } x _ { 4 } }
\end{array} \Leftrightarrow \left\{\begin{array}{l}
\left(\frac{4 E J}{L}+k_{1}\right) x_{2}+\frac{2 E J}{L} x_{4}=k_{1} \\
\frac{2 E J}{L} x_{2}+\left(\frac{4 E J}{L}+k_{2}\right) x_{4}=0 .
\end{array}\right.\right.
$$


Solving equations (3.3) to find $x_{2} ; x_{4}$ :

$$
\begin{aligned}
& x_{2}=\frac{\left(4 E J+k_{2} L\right) L k_{1}}{\left(12 E^{2} J^{2}+4 E J k_{2} L+4 L k_{1} E J+L^{2} k_{1} k_{2}\right)} ; \\
& x_{4}=\frac{-2 E J L k_{1}}{\left(12 E^{2} J^{2}+4 E J k_{2} L+4 L k_{1} E J+L^{2} k_{1} k_{2}\right)} ; \\
& {\left[\begin{array}{llll}
k_{12} & k_{22} & k_{32} & k_{42}
\end{array}\right]^{T}=\left[\begin{array}{lll}
k_{e}
\end{array}\right] \times\left[\begin{array}{llll}
x_{1} & x_{2} & x_{3} & x_{4}
\end{array}\right]^{T}}
\end{aligned}
$$

$$
\left[\begin{array}{l}
k_{12} \\
k_{22} \\
k_{32} \\
k_{42}
\end{array}\right]=\frac{E J}{L\left(12 E^{2} J^{2}+4 E J k_{2} L+4 L k_{1} E J+L^{2} k_{1} k_{2}\right)}\left[\begin{array}{c}
6\left(4 E J+k_{2} L\right) k_{1}-12 E J k_{1} \\
{\left[4\left(4 E J+k_{2} L\right) k_{1}-4 E J k_{1}\right] L} \\
-6\left(4 E J+k_{2} L\right) k_{1}+12 E J k_{1} \\
{\left[2\left(4 E J+k_{2} L\right) k_{1}-8 E J k_{1}\right] L}
\end{array}\right]
$$

The third column of matrix $\left[k_{e s}\right]$ is created as follows

Boundary conditions, Fig. 1e:

$$
\begin{aligned}
& \{X\}=\left[\begin{array}{llll}
X_{1} & X_{2} & X_{3} & X_{4}
\end{array}\right]^{T}=\left[\begin{array}{llll}
0 & 0 & 1 & 0
\end{array}\right]^{T} \\
& f_{2}=-k_{1} x_{2} ; \quad f_{4}=-k_{2} x_{4} ; \quad x_{1}=0, \quad x_{3}=1 .
\end{aligned}
$$

To impose the boundary conditions into equations (2.1) that becomes:

$$
\left\{\begin{array} { l } 
{ \frac { 4 E J } { L } x _ { 2 } - \frac { 6 E J } { L ^ { 2 } } x _ { 3 } + \frac { 2 E J } { L } x _ { 4 } = - k _ { 1 } x _ { 2 } } \\
{ \frac { 2 E L } { L } x _ { 2 } - \frac { 6 E J } { L ^ { 2 } } x _ { 3 } + \frac { 4 E J } { L } x _ { 4 } = - x _ { 4 } k _ { 2 } }
\end{array} \Leftrightarrow \left\{\begin{array}{l}
\left(\frac{4 E J}{L}+k_{1}\right) x_{2}+\frac{2 E J}{L} x_{4}=\frac{6 E J}{L^{2}} \\
\frac{2 E J}{L} x_{2}+\left(\frac{4 E J}{L}+k_{2}\right) x_{4}=\frac{6 E J}{L^{2}}
\end{array}\right.\right.
$$

Solving equations (3.4) to find $x_{2} ; x_{4}$ :

$$
\begin{gathered}
x_{2}=\frac{6 E J\left(2 E J+k_{2} L\right)}{\left(12 E^{2} J^{2}+4 E J k_{2} L+4 L k_{1} E J+L^{2} k_{1} k_{2}\right) L}, \\
x_{4}=\frac{6 E J\left(2 E J+k_{1} L\right)}{\left(12 E^{2} J^{2}+4 E J k_{2} L+4 L k_{1} E J+L^{2} k_{1} k_{2}\right) L}, \\
{\left[k_{13} k_{23} k_{33} k_{43}\right]^{T}=\left[k_{e}\right] \times\left[x_{1} x_{2} x_{3} x_{4}\right]^{T}} \\
{\left[\begin{array}{l}
k_{13} \\
k_{23} \\
k_{33} \\
k_{43}
\end{array}\right]=\left[\begin{array}{l}
\frac{36 E^{2} J^{2}\left(2 E J+k_{2} L\right)+36 E^{2} J^{2}\left(2 E J+k_{1} L\right)}{L^{3}\left(12 E^{2} J^{2}+4 E J k_{2} L+4 L k_{1} E J+L^{2} k_{1} k_{2}\right)}-\frac{12 E J}{L^{3}} \\
\frac{24 E^{2} J^{2}\left(2 E J+k_{2} L\right)+12 E J^{2}\left(2 E J+k_{1} L\right)}{L^{2}\left(12 E^{2} J^{2}+4 E J k_{2} L+4 L k_{1} E J+L^{2} k_{1} k_{2}\right)}-\frac{6 E J}{L^{2}} \\
\frac{-36 E^{2} J^{2}\left(2 E J+k_{2} L\right)-36 E^{2} J^{2}\left(2 E J+k_{1} L\right)}{L^{3}\left(12 E^{2} J^{2}+4 E J k_{2} L+4 L k_{1} E J+L^{2} k_{1} k_{2}\right)}+\frac{12 E J}{L^{3}} \\
\frac{12 E^{2} J^{2}\left(2 E J+k_{2} L\right)+24 E^{2} J^{2}\left(2 E J+k_{1} L\right)}{L^{2}\left(12 E^{2} J^{2}+4 E J k_{2} L+4 L k_{1} E J+L^{2} k_{1} k_{2}\right)}-\frac{6 E J}{L^{2}}
\end{array}\right]}
\end{gathered}
$$


The fourth column of matrix $\left[k_{e s}\right]$ is created as the follows

Boundary conditions, Fig. 1f:

$$
\begin{aligned}
& \{X\}=\left[\begin{array}{llll}
X_{1} & X_{2} & X_{3} & X_{4}
\end{array}\right]^{T}=\left[\begin{array}{llll}
0 & 0 & 0 & 1
\end{array}\right]^{T} \\
& f_{2}=-k_{1} x_{2} ; \quad f_{4}=k_{2}-k_{2} x_{4} ; \quad x_{1}=0, \quad x_{3}=0 .
\end{aligned}
$$

To impose the boundary conditions into equations (2.1) that becomes:

$$
\left\{\begin{array} { l } 
{ \frac { 4 E J } { L } x _ { 2 } + \frac { 2 E J } { L } x _ { 4 } = - k _ { 1 } x _ { 2 } } \\
{ \frac { 2 E J } { L } x _ { 2 } + \frac { 4 E J } { L } x _ { 4 } = k _ { 2 } - k _ { 2 } x _ { 4 } }
\end{array} \Leftrightarrow \left\{\begin{array}{l}
\left(\frac{4 E J}{L}+k_{1}\right) x_{2}+\frac{2 E J}{L} x_{4}=0 \\
\frac{2 E J}{L} x_{2}+\left(\frac{4 E J}{L}+k_{2}\right) x_{4}=k_{2}
\end{array}\right.\right.
$$

Solving equations (3.5) to find $x_{2}, x_{4}$ :

$$
\begin{aligned}
& x_{2}=\frac{-2 E J L k_{2}}{\left(12 E^{2} J^{2}+4 E J k_{2} L+4 L k_{1} E J+L^{2} k_{1} k_{2}\right.}, \\
& x_{4}=\frac{\left(4 E J+k_{1} L\right) L k_{2}}{\left(12 E^{2} J^{2}+4 E J k_{2} L+4 L k_{1} E J+L^{2} k_{1} k_{2}\right)} \\
& {\left[\begin{array}{llll}
k_{14} & k_{24} & k_{34} & k_{44}
\end{array}\right]^{T}=\left[\begin{array}{lll}
k_{e}
\end{array}\right] \times\left[\begin{array}{llll}
x_{1} & x_{2} & x_{3} & x_{4}
\end{array}\right]^{T}}
\end{aligned}
$$

$$
\left[\begin{array}{l}
k_{14} \\
k_{24} \\
k_{34} \\
k_{44}
\end{array}\right]=\frac{E J}{L\left(12 E^{2} J^{2}+4 E J k_{2} L+4 L k_{1} E J+L^{2} k_{1} k_{2}\right)}\left[\begin{array}{c}
6\left(4 E J+k_{1} L\right) k_{2}-12 E J k_{2} \\
{\left[2\left(4 E J+k_{1} L\right) k_{2}-8 E J k_{2}\right] L} \\
-6\left(4 E J+k_{1} L\right) k_{2}+12 E J k_{2} \\
{\left[\left(4 E J+k_{1} L\right) k_{2}-4 E J k_{2}\right] L}
\end{array}\right]
$$

\subsection{To create the geometric stiffness matrix of beam with semirigid} connections $\left[k_{\sigma s}\right]$

From equations (2.2) the equilibrium equations for beam with semirigid connections, are in matrix form, Fig. $1 \mathrm{~b}$, where: $\left[k_{\sigma s}\right]$ is the geometric stiffness matrix of beam with semirigid connections;

$$
\left[\begin{array}{l}
F_{1} \\
F_{2} \\
F_{3} \\
F_{4}
\end{array}\right]=\left[\begin{array}{llll}
k_{11} & k_{12} & k_{13} & k_{14} \\
k_{21} & k_{22} & k_{23} & k_{24} \\
k_{31} & k_{32} & k_{33} & k_{34} \\
k_{41} & k_{42} & k_{43} & k_{44}
\end{array}\right] \times\left[\begin{array}{c}
X_{1} \\
X_{2} \\
X_{3} \\
X_{4}
\end{array}\right] \quad \text { or }\{F\}=\left[k_{\sigma s}\right]\{X\}
$$

The equilibrium equations of nodal point forces:

$$
\begin{aligned}
& \{F\}=\{f\} \\
& \{F\}=\left[\begin{array}{llll}
F_{1} & F_{2} & F_{3} & F_{4}
\end{array}\right]^{T} ; \quad\{f\}=\left[\begin{array}{llll}
f_{1} & f_{2} & f_{3} & f_{4}
\end{array}\right]^{T}
\end{aligned}
$$

The first column of matrix $\left[k_{\sigma s}\right]$ is created as follows 
Boundary conditions, Fig. 1c:

$$
\begin{aligned}
& \left\{\begin{array}{l}
X\} \\
X
\end{array}\left[\begin{array}{llll}
X_{1} & X_{2} & X_{3} & X_{4}
\end{array}\right]^{T}=\left[\begin{array}{llll}
1 & 0 & 0 & 0
\end{array}\right]^{T}\right. \\
& f_{2}=-k_{1} x_{2} ; \quad f_{4}=-k_{2} x_{4} ; \quad x_{1}=1, \quad x_{3}=0 .
\end{aligned}
$$

To impose the boundary conditions into equations (2.2) that becomes:

$$
\left\{\begin{array} { l } 
{ \frac { P } { 1 0 } x _ { 1 } + \frac { 2 P L } { 1 5 } x _ { 2 } - \frac { P L } { 3 0 } x _ { 4 } = - k _ { 1 } x _ { 2 } } \\
{ \frac { P } { 1 0 } x _ { 1 } - \frac { P L } { 3 0 } x _ { 2 } + \frac { 2 P L } { 1 5 } x _ { 4 } = - k _ { 2 } x _ { 4 } }
\end{array} \Leftrightarrow \left\{\begin{array}{l}
\left(\frac{2 P L}{15}+k_{1}\right) x_{2}-\frac{P L}{30} x_{4}=-\frac{P}{10} \\
-\frac{P L}{30} x_{2}+\left(\frac{2 P L}{15}+k_{2}\right) x_{4}=-\frac{P}{10}
\end{array}\right.\right.
$$

Solving equations (3.7) to find $x_{2} ; x_{4}$ :

$$
\begin{gathered}
x_{2}=-\frac{P\left(P L+6 k_{2}\right)}{P^{2} L^{2}+8 k_{2} P L+8 k_{1} P L+60 k_{1} k_{2}}, \\
x_{4}=-\frac{P\left(P L+6 k_{1}\right)}{P^{2} L^{2}+8 k_{2} P L+8 k_{1} P L+60 k_{1} k_{2}}, \\
{\left[\begin{array}{ll}
\left.k_{11} k_{21} k_{31} k_{41}\right]^{T}=\left[k_{\sigma}\right] \times\left[x_{1} x_{2} x_{3} x_{4}\right]^{T} \\
{\left[\begin{array}{l}
k_{11} \\
k_{21} \\
k_{31} \\
k_{41}
\end{array}\right]=\left[\begin{array}{l}
\frac{6 P}{5 L}-\frac{P^{2}\left(P L+6 k_{2}\right)+P^{2}\left(P L+6 k_{1}\right)}{10\left(P^{2} L^{2}+8 k_{2} P L+8 k_{1} P L+60 k_{1} k_{2}\right)} \\
\frac{P}{10}-\frac{4 P^{2} L\left(P L+6 k_{2}\right)-P^{2} L\left(P L+6 k_{1}\right)}{30\left(P^{2} L^{2}+8 k_{2} P L+8 k_{1} P L+60 k_{1} k_{2}\right)} \\
-\frac{6 P}{5 L}+\frac{P^{2}\left(P L+6 k_{2}\right)+P^{2}\left(P L+6 k_{1}\right)}{10\left(P^{2} L^{2}+8 k_{2} P L+8 k_{1} P L+60 k_{1} k_{2}\right)} \\
\frac{P}{10}+\frac{P^{2} L\left(P L+6 k_{2}\right)-4 P^{2} L\left(P L+6 k_{1}\right)}{30\left(P^{2} L^{2}+8 k_{2} P L+8 k_{1} P L+60 k_{1} k_{2}\right)}
\end{array}\right]}
\end{array}\right.}
\end{gathered}
$$

The second column of matrix $\left[k_{\sigma s}\right]$ is created as follows

Boundary conditions, Fig. 1d:

$$
\begin{aligned}
& \left\{\begin{array}{l}
X\} \\
\}
\end{array}\left[\begin{array}{lllll}
X_{1} & X_{2} & X_{3} & X_{4}
\end{array}\right]^{T}=\left[\begin{array}{llll}
0 & 1 & 0 & 0
\end{array}\right]^{T}\right. \\
& f_{2}=k_{1}-k_{1} x_{2} ; \quad f_{4}=-k_{2} x_{4} ; \quad x_{1}=0, \quad x_{3}=0 .
\end{aligned}
$$

To impose the boundary conditions into equations (2.2) that becomes:

$$
\left\{\begin{array} { l } 
{ \frac { 2 P L } { 1 5 } x _ { 2 } - \frac { P L } { 3 0 } x _ { 4 } = k _ { 1 } - k _ { 1 } x _ { 2 } } \\
{ - \frac { P L } { 3 0 } x _ { 2 } + \frac { 2 P L } { 1 5 } x _ { 4 } = - k _ { 2 } x _ { 4 } }
\end{array} \Leftrightarrow \left\{\begin{array}{l}
\left(\frac{2 P L}{15}+k_{1}\right) x_{2}-\frac{P L}{30} x_{4}=k_{1} \\
-\frac{P L}{30} x_{2}+\left(\frac{2 P L}{15}+k_{2}\right) x_{4}=0
\end{array}\right.\right.
$$


Solving equations (3.8) to find $x_{2}, x_{4}$ :

$$
\begin{gathered}
x_{2}=\frac{4 k_{1}\left(2 P L+15 k_{2}\right)}{P^{2} L^{2}+8 k_{2} P L+8 k_{1} P L+60 k_{1} k_{2}} ; \\
x_{4}=\frac{2 P L k_{1}}{P^{2} L^{2}+8 k_{2} P L+8 k_{1} P L+60 k_{1} k_{2}} ; \\
{\left[\begin{array}{lll}
k_{12} & k_{22} k_{32} k_{42}
\end{array}\right]^{T}=\left[k_{\sigma}\right] \times\left[x_{1} x_{2} x_{3} x_{4}\right]^{T}} \\
{\left[\begin{array}{l}
k_{12} \\
k_{22} \\
k_{32} \\
k_{42}
\end{array}\right]=\frac{P}{\left(P^{2} L^{2}+8 k_{2} P L+8 k_{1} P L+60 k_{1} k_{2}\right)}\left[\begin{array}{c}
P L k_{1}+6 P k_{1} k_{2} \\
P L k_{1}+8 L k_{1} k_{2} \\
-P k_{1} L-6 k_{1} k_{2} \\
-2 L k_{1} k_{2}
\end{array}\right]}
\end{gathered}
$$

The third column of matrix $\left[k_{\sigma s}\right]$ is created as follows

Boundary conditions, Fig. Ie:

$$
\begin{aligned}
& \{X\}=\left[\begin{array}{llll}
X_{1} & X_{2} & X_{3} & X_{4}
\end{array}\right]^{T}=\left[\begin{array}{llll}
0 & 0 & 1 & 0
\end{array}\right]^{T} \\
& f_{2}=-k_{1} x_{2} ; \quad f_{4}=-k_{2} x_{4} ; \quad x_{1}=0, \quad x_{3}=1 .
\end{aligned}
$$

To impose the boundary conditions into equations (2.2) that becomes

$$
\left\{\begin{array} { l } 
{ \frac { 2 P L } { 1 5 } x _ { 2 } - \frac { P } { 1 0 } x _ { 3 } - \frac { P L } { 3 0 } x _ { 4 } = - k _ { 1 } x _ { 2 } } \\
{ - \frac { P L } { 3 0 } x _ { 2 } - \frac { P } { 1 0 } x _ { 3 } + \frac { 2 P L } { 1 5 } x _ { 4 } = - k _ { 2 } x _ { 4 } }
\end{array} \Leftrightarrow \left\{\begin{array}{l}
\left(\frac{2 P L}{15}+k_{1}\right) x_{2}-\frac{P L}{30} x_{4}=\frac{P}{10} \\
-\frac{P L}{30} x_{2}+\left(\frac{2 P L}{15}+k_{2}\right) x_{4}=\frac{P}{10}
\end{array}\right.\right.
$$

Solving equations (3.9) to find $x_{2}, x_{4}$ :

$$
\begin{gathered}
x_{2}=\frac{P\left(P L+6 k_{2}\right)}{P^{2} L^{2}+8 k_{2} P L+8 k_{1} P L+60 k_{1} k_{2}} ; \\
x_{4}=\frac{P\left(P L+6 k_{1}\right)}{P^{2} L^{2}+8 k_{2} P L+8 k_{1} P L+60 k_{1} k_{2}}, \\
{\left[k_{13} k_{23} k_{33} k_{43}\right]^{T}=\left[k_{\sigma}\right] \times\left[x_{1} x_{2} x_{3} x_{4}\right]^{T}} \\
{\left[\begin{array}{l}
k_{13} \\
k_{23} \\
k_{33} \\
k_{43}
\end{array}\right]=\left[\begin{array}{l}
-\frac{6 P}{5 L}+\frac{P^{2}\left(P L+6 k_{2}\right)+P^{2}\left(P L+6 k_{1}\right)}{10\left(P^{2} L^{2}+8 k_{2} P L+8 k_{1} P L+60 k_{1} k_{2}\right)} \\
-\frac{P}{10}+\frac{4 P^{2} L\left(P L+6 k_{2}\right)-P^{2} L\left(P L+6 k_{1}\right)}{30\left(P^{2} L^{2}+8 k_{2} P L+8 k_{1} P L+60 k_{1} k_{2}\right)} \\
\frac{P^{2}}{5 L}-\frac{P^{2}\left(P L+6 k_{2}\right)+P^{2}\left(P L+6 k_{1}\right)}{10\left(P^{2} L^{2}+8 k_{2} P L+8 k_{1} P L+60 k_{1} k_{2}\right)} \\
-\frac{P}{10}-\frac{P^{2} L\left(P L+6 k_{2}\right)-4 P^{2} L\left(P L+6 k_{1}\right)}{30\left(P^{2} L^{2}+8 k_{2} P L+8 k_{1} P L+60 k_{1} k_{2}\right)}
\end{array}\right]}
\end{gathered}
$$


The fourth column of matrix $\left[k_{\sigma s}\right]$ is created as follows

Boundary conditions, Fig. 1f:

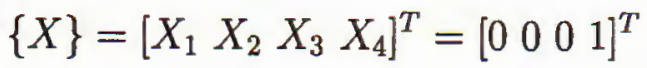

$$
\begin{aligned}
& f_{2}=-k_{1} x_{2} ; \quad f_{4}=-k_{2} x_{4} ; \quad x_{1}=0, \quad x_{3}=0 .
\end{aligned}
$$

To impose the boundary conditions into equations (2.2) that becomes:

$$
\left\{\begin{array} { l } 
{ \frac { 2 P L } { 1 5 } x _ { 2 } - \frac { P L } { 3 0 } x _ { 4 } = - k _ { 1 } x _ { 2 } } \\
{ - \frac { P L } { 3 0 } x _ { 2 } + \frac { 2 P L } { 1 5 } x _ { 4 } = k _ { 2 } X _ { 4 } - k _ { 2 } x _ { 4 } }
\end{array} \Leftrightarrow \left\{\begin{array}{l}
\left(\frac{2 P L}{15}+k_{1}\right) x_{2}-\frac{P L}{30} x_{4}=0 \\
-\frac{P L}{30} x_{2}+\left(\frac{2 P L}{15}+k_{2}\right) x_{4}=k_{2}
\end{array}\right.\right.
$$

Solving equations $(3.10)$ to find $x_{2}, x_{4}$ :

$$
\begin{aligned}
& x_{2}=\frac{2 P L k_{2}}{P^{2} L^{2}+8 k_{2} P L+8 k_{1} P L+60 k_{1} k_{2}}, \\
& x_{4}=\frac{4 k_{2}\left(2 P L+15 k_{1}\right)}{P^{2} L^{2}+8 k_{2} P L+8 k_{1} P L+60 k_{1} k_{2}}, \\
& {\left[\begin{array}{llll}
k_{14} & k_{24} & k_{34} & k_{44}
\end{array}\right]^{T}=\left[\begin{array}{ll}
k_{\sigma}
\end{array}\right] \times\left[\begin{array}{llll}
x_{1} & x_{2} & x_{3} & x_{4}
\end{array}\right]^{T} .} \\
& {\left[\begin{array}{l}
k_{14} \\
k_{24} \\
k_{34} \\
k_{44}
\end{array}\right]=\frac{P}{\left(P^{2} L^{2}+8 k_{2} P L+8 k_{1} P L+60 k_{1} k_{2}\right)}\left[\begin{array}{c}
P L k_{2}+6 k_{1} k_{2} \\
-2 L k_{1} k_{2} \\
-P k_{2} L-6 k_{1} k_{2} \\
P L^{2} k_{2}+8 L k_{1} k_{2}
\end{array}\right]}
\end{aligned}
$$

4. To create the stiffness matrix and geometric stiffness matrix of beam with semirigid connections and rigidzones (or rigid offset) $\left[k_{e s}^{*}\right]$; $\left[k_{\sigma s}^{*}\right]$ - Plane frame

Equilibrium equations for vector of nodal point forces for $e_{1}$ and $e_{2}$, Fig. $3 \mathrm{~b}$. form

$F_{1}=F_{1}^{*} ; \quad F_{2}=F_{2}^{*}-F_{1}^{*} e_{1} ; \quad F_{3}=F_{3}^{*} ; \quad F_{4}=F_{4}^{*}+F_{3}^{*} e_{2}$, are written in matrix

$$
\left[\begin{array}{l}
F_{1} \\
F_{2} \\
F_{3} \\
F_{4}
\end{array}\right]=\left[\begin{array}{cccc}
1 & 0 & 0 & 0 \\
-e_{1} & 1 & 0 & 0 \\
0 & 0 & 1 & 0 \\
0 & 0 & e_{2} & 1
\end{array}\right] \times\left[\begin{array}{c}
F_{1}^{*} \\
F_{2}^{*} \\
F_{3}^{*} \\
F_{4}^{*}
\end{array}\right] \text { or }\{F\}=\left[E_{1}\right]\left\{F^{*}\right\}
$$

Equilibrium equations for vector of nodal point displacements for $e_{1}$ and $e_{2}$, Fig. 3b. $X_{1}=X_{1}^{*}+X_{2}^{*} e_{1} ; X_{2}=X_{2}^{*} ; X_{3}=X_{3}^{*}-X_{4}^{*} e_{2} ; X_{4}=X_{4}^{*}$, are written in matrix form:

$$
\left[\begin{array}{l}
X_{1} \\
X_{2} \\
X_{3} \\
X_{4}
\end{array}\right]=\left[\begin{array}{cccc}
1 & e_{1} & 0 & 0 \\
0 & 1 & 0 & 0 \\
0 & 0 & 1 & -e_{2} \\
0 & 0 & 0 & 1
\end{array}\right] \times\left[\begin{array}{c}
X_{1}^{*} \\
X_{2}^{*} \\
X_{3}^{*} \\
X_{4}^{*}
\end{array}\right] \quad \text { or }\{X\}=\left[E_{2}\right]\left\{X^{*}\right\}
$$


and $\left[E_{1}\right]^{-1}=\left[E_{2}\right]^{T}$.

Substituted (4.1), (4.2) into equations $\{F\}=\left[k_{e s}\right]\{X\}$ that becomes:

$\left[E_{1}\right]\left\{F^{*}\right\}=\left[k_{e s}\right] \cdot\left[E_{2}\right] \cdot\left\{X^{*}\right\} \Leftrightarrow\left\{F^{*}\right\}=\left[E_{1}\right]^{-1}\left[k_{e s}\right] \cdot\left[E_{2}\right] \cdot\left\{X^{*}\right\} \Leftrightarrow\left\{F^{*}\right\}=$ $\left[E_{2}\right]^{T} \cdot\left[k_{e s}\right] \cdot\left[E_{2}\right] \cdot\left\{X^{*}\right\}$.

So that $\left[k_{e s}^{*}\right]=\left[E_{2}\right]^{T}\left[k_{e s}\right] \cdot\left[E_{2}\right]$ and similar $\left[k_{\sigma s}^{*}\right]=\left[E_{2}\right]^{T}\left[k_{\sigma s}\right] \cdot\left[E_{2}\right]$.

5. To create vector of nodal point loads of beam with semirigid connections

5.1 Vector of nodal point loads for uniform load of beam with semirigid connections $\left\{q_{e m}\right\}$, Fig. 2a

$$
\left\{q_{e}\right\}=\left[k_{e}\right]\{x\},
$$

where

$$
\left\{q_{e}\right\}=\left[\begin{array}{llll}
\frac{q L}{2} & \frac{q L^{2}}{12} & \frac{q L}{2} & -\frac{q L^{2}}{12}
\end{array}\right]^{T} .
$$

To impose the boundary conditions $\left(x_{1}=0, x_{3}=0\right)$ into equations (5.1) that becomes:

$$
\left\{\begin{array} { l } 
{ \frac { 4 E J } { L } x _ { 2 } + \frac { 2 E J } { L } x _ { 4 } = \frac { q L ^ { 2 } } { 1 2 } - k _ { 1 } x _ { 2 } } \\
{ \frac { 2 E J } { L } x _ { 2 } + \frac { 4 E J } { L } x _ { 4 } = - \frac { q L ^ { 2 } } { 1 2 } - k _ { 2 } x _ { 4 } }
\end{array} \Leftrightarrow \left\{\begin{array}{l}
\left(\frac{4 E J}{L}+k_{1}\right) x_{2}+\frac{2 E J}{L} x_{4}=\frac{q L^{2}}{12} \\
\frac{2 E J}{L} x_{2}+\left(\frac{4 E J}{L}+k_{2}\right) x_{4}=-\frac{q L^{2}}{12}
\end{array}\right.\right.
$$

Solving equations (5.2) to find $x_{2}, x_{4}$ :

$$
\begin{aligned}
& x_{2}=\frac{q L^{3}\left(6 E J+k_{2} L\right)}{12\left(12 E^{2} J^{2}+4 k_{2} L E J+4 k_{1} L E J+k_{1} L^{2} k_{2}\right)}, \\
& x_{4}=-\frac{q L^{3}\left(6 E J+k_{1} L\right)}{12\left(12 E^{2} J^{2}+4 k_{2} L E J+4 k_{1} L E J+k_{1} L^{2} k_{2}\right)} \\
& \left\{q_{e m}\right\}=\left\{q_{e}\right\}-\left[k_{e}\right]\{x\}
\end{aligned}
$$

5.2 Vector of nodal point loads for concentrated load at middle of beam with semirigid connections $\left\{P_{e m}\right\}$, Fig. $2 \mathrm{~b}$

$$
\left\{P_{e}\right\}=\left[k_{e}\right]\{x\}
$$

where

$$
\left\{P_{e}\right\}=\left[\begin{array}{llll}
\frac{P L}{2} & \frac{P L^{2}}{8} & \frac{P L}{2} & -\frac{P L^{2}}{8}
\end{array}\right]^{T} .
$$


To impose the boundary conditions $\left(x_{1}=0, x_{3}=0\right)$ into equations (5.4) that becomes:

$$
\left\{\begin{array} { l } 
{ \frac { 4 E J } { L } x _ { 2 } + \frac { 2 E J } { L } x _ { 4 } = \frac { P L ^ { 2 } } { 8 } - k _ { 1 } x _ { 2 } } \\
{ \frac { 2 E J } { L } x _ { 2 } + \frac { 4 E J } { L } x _ { 4 } = - \frac { P L ^ { 2 } } { 8 } - k _ { 2 } x _ { 4 } }
\end{array} \Leftrightarrow \left\{\begin{array}{l}
\left(\frac{4 E J}{L}+k_{1}\right) x_{2}+\frac{2 E J}{L} x_{4}=\frac{P L^{2}}{8} \\
\frac{2 E J}{L} x_{2}+\left(\frac{4 E J}{L}+k_{2}\right) x_{4}=-\frac{P L^{2}}{8}
\end{array}\right.\right.
$$

Solving equations (5.5) to find $x_{2}, x_{4}$ :

$$
\begin{aligned}
& x_{2}=\frac{1}{8} \frac{P L^{3}\left(6 E J+k_{2} L\right)}{\left(12 E^{2} J^{2}+4 k_{2} L E J+4 k_{1} L E J+k_{1} k_{2} L^{2}\right)} \\
& x_{4}=-\frac{1}{8} \frac{P L^{3}\left(6 E J+k_{1} L\right)}{\left(12 E^{2} J^{2}+4 k_{2} L E J+4 k_{1} L E J+k_{1} k_{2} L^{2}\right)} \\
& \left\{P_{e m}\right\}=\left\{P_{e}\right\}-\left[k_{e}\right]\{x\}
\end{aligned}
$$

\section{Numerical analysis}

The structural stiffness equations are formed by superimposing the member stiffness include effects of geometric nonlinearity and connection flexibility. The structure matrix $\left[K_{\sigma s}\right]$ is built by summing overlapping terms of element matrices $\left[k_{\sigma s}\right]$, in the same way that the conventional $\left[K_{e s}\right]$ is built by summing overlapping terms of element matrices $\left[k_{e s}\right]$.

$$
\left(\left[K_{e s}\right]+\lambda_{c r}\left[K_{o s}\right]_{r e f}\right)\{d X\}=\{0\} .
$$

Equation (6.1) defines an eigenvalue problem whose lowest eigenvalue $\lambda_{c r}$ is associated with buckling of frames with semirigid connections. The critical of buckling load is

$$
\{F\}_{c r}=\lambda_{c r}\{F\}_{\text {ref }}
$$

A column is an integral part of a frame, where end conditions depend on stiffness of the beams and girders framing into the column, and the rigidity of the beam column connections. An effective length factor $\mu$ is used to modify the actual length of the column for design purposes. The factor $\mu$ is defined as:

$$
\mu=\frac{\pi}{L} \sqrt{\frac{E J}{P_{c r}}} \cdots(\text { see }[4])
$$

\section{Comprehensive examples}

The develop analysis was numerically implemented through the computer program written by Pascal languge, which was tested for efficiency and reliability. 
Table 1. $\mu$ Factor - Comparison results between program and VSD Fig. 4a,b

\begin{tabular}{|c|c|c|c|c|c|c|c|c|c|}
\hline \multirow{2}{*}{$\begin{array}{l}\text { Pin-Pin } \\
\text { solution } \\
\text { VSD }\end{array}$} & \multicolumn{8}{|c|}{ Semrigid Solution Program - Joint Stiffness $\alpha=\frac{k}{i_{x}}$} & \multirow{2}{*}{$\begin{array}{l}\text { - Fixed-Fixed } \\
\text { solution } \\
\text { VSD }\end{array}$} \\
\hline & 0.001 & 0.01 & 0.1 & 1 & 10 & 100 & 1000 & $10 \mathrm{E} 4$ & \\
\hline - & - & - & - & - & - & - & - & - & - \\
\hline- & - & 35.54 & 10.717 & 4.627 & 3.572 & 3.436 & 3.436 & 3.436 & 3.42 \\
\hline- & - & 35.54 & 10.717 & 4.342 & 3.154 & 3.015 & 3.015 & 3.015 & 3.00 \\
\hline- & - & 35.54 & 10.717 & 4.051 & 2.801 & 2.657 & 2.642 & 2.627 & 2.63 \\
\hline- & - & 35.54 & 9.858 & 3.855 & 2.507 & 2.349 & 2.329 & 2.329 & 2.33 \\
\hline- & - & 35.54 & 9.858 & 3.767 & 2.359 & 2.183 & 2.159 & 2.159 & 2.17 \\
\hline- & - & 35.54 & 9.858 & 3.726 & 2.299 & 2.128 & 2.105 & 2.105 & 2.11 \\
\hline - & - & 35.54 & 9.858 & 3.686 & 2.226 & 2.049 & 2.049 & 2.029 & 2.00 \\
\hline
\end{tabular}

Table 2. $\mu$ Factor-Comparison results between program and VSD Fig. 5a,b,c $\frac{i_{x}}{i_{c}}$ Pin-Pin Semrigid Solution Program - Joint Stiffness $\alpha=\frac{k}{i_{x}}$ Fixed-Fixed

\begin{tabular}{|c|c|c|c|c|c|c|c|c|c|}
\hline \multicolumn{9}{|c|}{ solution } & \multirow{2}{*}{$\begin{array}{l}\text { solution } \\
\text { VSD }\end{array}$} \\
\hline VSD & 0.001 & 0.01 & 0.1 & 1 & 10 & 100 & 1000 & $10 \mathrm{E} 4$ & \\
\hline 2 & 2 & 2 & 2 & 2 & 2 & 2 & 2 & 2 & 2 \\
\hline 2 & 2 & 1.999 & 1.924 & 1.685 & 1.522 & 1.495 & 1.495 & 1.495 & 1.50 \\
\hline 2 & 2 & 1.984 & 1.919 & 1.648 & 1.433 & 1.397 & 1.393 & 1.393 & 1.40 \\
\hline 2 & 2 & 1.984 & 1.919 & 1.611 & 1.331 & 1.280 & 1.273 & 1.273 & 1.28 \\
\hline 2 & 2 & 1.984 & 1.919 & 1.579 & 1.228 & 1.160 & 1.152 & 1.151 & 1.16 \\
\hline 2 & 2 & 1.984 & 1.919 & 1.560 & 1.165 & 1.086 & 1.078 & 1.077 & 1.08 \\
\hline 2 & 2 & 1.984 & 1.919 & 1.554 & 1.142 & 1.060 & 1.050 & 1.050 & 1.06 \\
\hline 2 & 2 & 1.984 & 1.919 & 1.545 & 1.108 & 1.020 & 1.011 & 1.010 & 1.00 \\
\hline
\end{tabular}

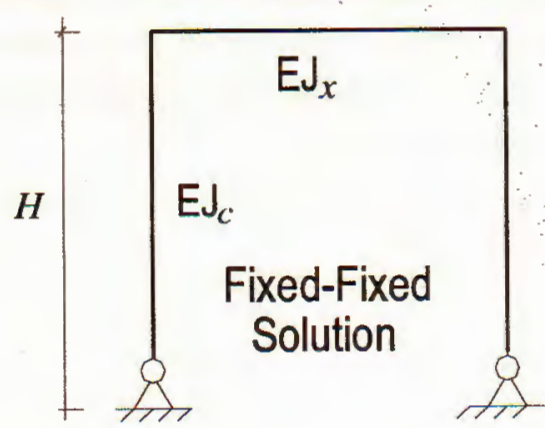

(4.a)

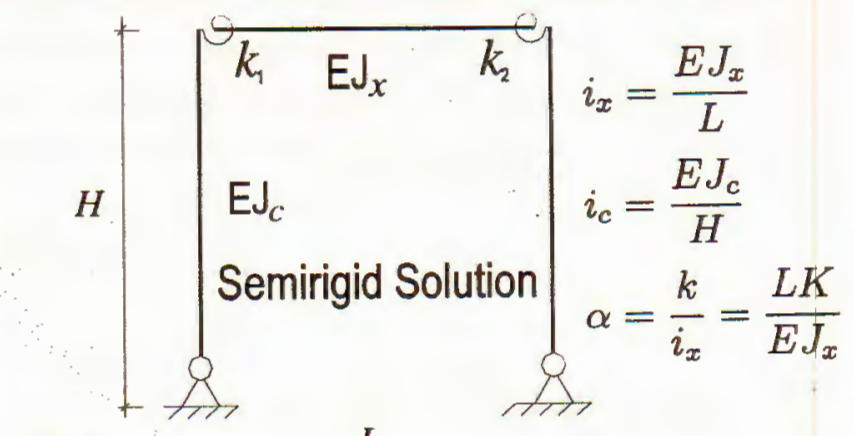

(4.b)

Fig. 4 

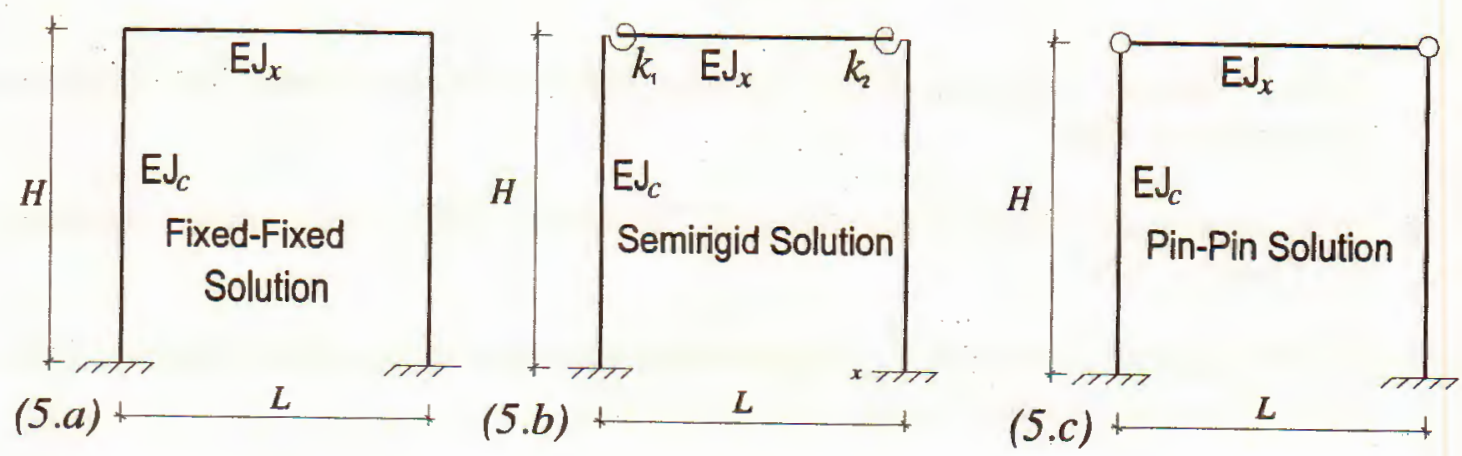

Fig. 5

The input is methodically and economically grouped into data files with very little time and effort required for their preparation. Most features broadening the scope of the analysis have been transferred to the computer code. Several plane frames are investigated using the program to demonstrate the validity and effectiveness of the stability analysis for semirigid frames. The table 1 and table 2 illustrated for $i_{x} / i_{c}=0$ to 10 and $\alpha=0.001$ to $\alpha=10 E 4$ respectively with simple connections and rigid connections, for semirigid connections $k_{1}=k_{2}=k$.

\section{Conclusions}

The first-and second-order stiffness matrices of beam-column of double symmetrical cross section with semirigid connections and semirigid connections with rigidzones including the effects of end axial loads are derived in a classical manner. The derived matrices can be used in the stability, first- and second-order elastic analyses of framed structures with rigid, semirigid, and simple connections with or without rigidzones. The proposed method uses the axial loads in columns obtained from a first-order linear analysis to determine the critical axial load, effective length $\mu$ factor of each column of framed structure in any type of construction.

The advantages of the proposed method are: (1) the effects of semirigid connections are condensed into the stiffness and geometric stiffness matrix coefficients without introducing additional degrees of freedom; and (2) both matrices can be incorporated into computer programs without major difficulties.

In current engineering practice, the determination of the $\mu$ factor of columns must be made in order to design frames based on VSD, [2]. In the present paper, the governing equations for determining the column $\mu$ factor for frames with rigid, semirigid, and simple connections and rigidzones under various boundary conditions are derived based on the stability of semirigid frames. The validity of both matrices is verified against available solutions of stability analysis, table 1, 2 and VSD [2].

\section{REFERENCES}

1. Cook R. D., Malkus D. S. and Plesha M. E. Concepts and Applications of 
Finite Element Analysis, Third Edition, John Wiley and Sons, Inc, ISBN 0471-84788-7, 1989

2. Steel structures - Design Standard. TCVN 5575: 1991-(Vietnamese Standard for Design - VSD)

3. Ariaratnam S. T. and Xu L. Uniqueness in analysis of semirigid Frames, Journal of Structural Engineering - January 1996.

4. Mukhanov K. K. Design of Metal Structures, Mir Publishers, Moscow 1968.

5. Richard H. Gallagher. Finite Element Analysis Fundamental, Prentice-Hall,INC., Englewood Cliffs, New Jersey -1975.

6. Abraham J. Rokack, MSCE. Theory and Problems of Structural Steel Design (Load and Resistance Factor Design Specification for Structural Steel Buildings -American Institute of Steel Construction), Schaum's Outline Series, McGraw-Hill, INC., New-York 1991.

7. Gerardy J.-C. C.E.C. Agreement No 7210-Sa/507 Semi - Rigid Action in Steel Frame Structures, Service Recherches et Promotion Technique Structures ARBED - Recherches - 66, Rue de Luxembourg, L-4221 Esch/Alzette Luxembourg.

8. Patrick J. Dowling, John E. Harding, Reidar Bjorhovde. Construction Steel Design - An International Guide, Elsevier Applied Science London and NewYork 1985.

Received July 2, 2001

\section{PHÂN TÍCH ỔN ĐINHH KHUNG THÉP CÓ LIÊN KẾT MỀM VÀ KHUNG THÉP CÓ NÚT CỨNG CÙNG LIÊN KẾT MỀM}

Bài báo này trình bày cách thiết lập ma trận độ cứng, ma trận độ cứng hình hoc cho phần tử dầm liên kết đàn hồi tại hai đầu và phần tử dầm có nút cứng cùng liên kết dàn hồi tại hai đầu. Với hai ma trận được thiết lập có thể ứng dụng để phân tích ổn định của khung thép có liên kết cứng, liên kết mềm, và liên kết khớp có nút cứng hoặc không có nút cứng. 